$\begin{array}{cccc}\begin{array}{c}\text { Laboratory } \\ \text { number }\end{array} & \begin{array}{c}\text { Published } \\ \text { reference }\end{array} & \begin{array}{c}\text { Original date or } \\ \text { other value }\end{array} & \begin{array}{c}\text { Corrected date } \\ \text { or other value }\end{array} \\ -146 & 49: 148 & 1170 \pm 100 & \\ -147 & 49: 148 & 700 \pm 100 & \\ -148 & 49: 148 & 1300 \pm 100 & \\ -150 & 49: 148 & 800 \pm 100 & \\ -151 & 50: 920 & 3450 \pm 120 & \\ -157 & 49: 149 & 4950 \pm 130 & \\ -169 & 49: 149 & 6300 \pm 150 & \\ -170 & 49: 149 & 6230 \pm 150 & \\ -171 & 49: 149 & 7100 \pm 160 & \\ -235 & 49: 149 & >37,000 & \\ -248 & 49: 149 & >37,000 & \\ -261^{2} & 50: 922 & >35,000\end{array}$

A.D./

B.C. date

A.D. 780

A.D. 1250

A.D. 650

A.D. 1150

1500 B.C.

3000 B.C.

4350 B.C.

4280 B.C.

5150 B.C.

${ }^{2}$ Modern standard, for terrestrial organic matter and for shallow-water marine carbonate, was age-corrected pre-industrial wood, probably not significantly different from $95 \%$ of NBS oxalic-acid standard. Contemporary assay of age-corrected pre-industrial marine shell from the same region was not significantly different from that of wood, and carbonate samples are therefore believed not to require correction for isotopic fractionation.

${ }^{2}$ Carbonate sample, not corrected for isotopic fractionation.

\title{
Ohio Wesleyan University
}

\begin{tabular}{|c|c|c|c|c|c|}
\hline $\begin{array}{l}\text { Laboratory } \\
\text { number }\end{array}$ & $\begin{array}{l}\text { Published } \\
\text { reference }\end{array}$ & $\begin{array}{l}\text { Original date or } \\
\quad \text { other value }\end{array}$ & $\begin{array}{l}\text { Corrected date } \\
\text { or other value }\end{array}$ & $\partial \mathrm{C}^{14}, \%$ & $\begin{array}{l}\text { A.D./ } \\
\text { B.C. date }\end{array}$ \\
\hline OWU-6 ${ }^{1}$ & $148: 342$ & $11,352 \pm 211$ & & $243.4 \pm 23.7$ & 9402 B.C. \\
\hline$-8^{2}$ & $148: 342$ & $19,906 \pm 691$ & & $84.0 \pm 85.8$ & 17,956 B.C. \\
\hline$-10^{3}$ & $148: 343$ & $6756 \pm 111$ & & $431.3 \pm 14.0$ & 4806 B.C. \\
\hline-14 & $148: 343$ & $8075 \pm 103$ & & $366.0 \pm 12.8$ & 6125 B.C. \\
\hline$-16^{4}$ & $148: 343$ & $5394 \pm 152$ & & $511.0 \pm 19.0$ & 3444 B.C. \\
\hline$-17^{5}$ & $148: 343$ & $7040 \pm 291$ & & $416.3 \pm 36.4$ & 5090 B.C. \\
\hline$-19^{6}$ & $148: 343$ & $1904 \pm 84$ & & $789.6 \pm 10.4$ & A.D. 46 \\
\hline-21 & $148: 344$ & $\partial \mathrm{C}^{14}+20 \%$ & $44.5 \pm 1.7 \%$ c & $1444.5 \pm 17.1$ & \\
\hline-22 & $148: 344$ & $10,906 \pm 112$ & & $257.3 \pm 13.9$ & 8956 B.C. \\
\hline-27 & $148: 344$ & $8108 \pm 105$ & & $364.5 \pm 13.2$ & 6158 B.C. \\
\hline-28 & $148: 344$ & $9482 \pm 319$ & & $307.2 \pm 39.6$ & 7532 B.C. \\
\hline-30 & $148: 346$ & $1186 \pm 161$ & & $861.9 \pm 20.1$ & A.D. 764 \\
\hline-31 & $161: 169$ & $820 \pm 84$ & & $902.9 \pm 10.5$ & A.D. 1130 \\
\hline-33 & $148: 344$ & $4003 \pm 97$ & & $607.6 \pm 12.2$ & 2053 B.C. \\
\hline-34 & $148: 345$ & $6539 \pm 97$ & & $443.1 \pm 12.6$ & 4589 B.C. \\
\hline-38 & $148: 347$ & $106 \pm 100^{9}$ & & $850.0 \pm 13.6$ & A.D. 1844 \\
\hline-39 & $148: 347$ & $9588 \pm 210^{9}$ & & $261.1 \pm 25.1$ & 7638 B.C. \\
\hline$-42^{7}$ & $148: 345$ & $18,899 \pm 270$ & & $95.1 \pm 33.9$ & 16,9:9 B.C. \\
\hline-46 & $161: 169$ & $325 \pm 92^{\circ}$ & & $8271 \pm 11.5$ & A.D. 1625 \\
\hline-47 & $148: 347$ & $418 \pm 201^{9}$ & & $817.2 \pm 25.0$ & A.D. 1532 \\
\hline-48 & $161: 169$ & $950 \pm 260^{9}$ & & $764.9 \pm 33.3$ & A.D. 1000 \\
\hline-51 & $148: 345$ & $1772 \pm 53$ & & $802.5 \pm 6.5$ & A.D. 178 \\
\hline$-52^{8}$ & $148: 345$ & $17,880 \pm 224$ & & $108.0 \pm 31.1$ & 15,930 B.C. \\
\hline-53 & $148: 345$ & $10,558 \pm 365$ & & $268.8 \pm 45.6$ & 8608 B.C. \\
\hline-55 & $161: 169$ & $920 \pm 184^{9}$ & & $767.9 \pm 23.3$ & A.D. 1030 \\
\hline-57 & $148: 347$ & $3833 \pm 217^{9}$ & & $534.6 \pm 14.4$ & 1883 B.C. \\
\hline-58 & $148: 347$ & $4095 \pm 232^{9}$ & & $516.3 \pm 14.9$ & 2145 B.C. \\
\hline .59 & $148: 345$ & $3424 \pm 185$ & & $653.2 \pm 23.8$ & 1474 B.C. \\
\hline-60 & $148: 346$ & $2946 \pm 185$ & & $693.1 \pm 23.6$ & 996 B.C. \\
\hline-61 & $148: 346$ & $1469 \pm 65$ & & $832.9 \pm 8.1$ & A.D. 481 \\
\hline-62 & $148: 346$ & $1515 \pm 166$ & & $828.1 \pm 20.6$ & A.D. 435 \\
\hline$-71^{10}$ & $148: 347$ & $23,382 \pm 400$ & & $54.5 \pm 49.0$ & 21,432 B.C. \\
\hline-72 & $148: 346$ & $829 \pm 62$ & & $902.3 \pm 7.8$ & A.D. 1121 \\
\hline$-72 \mathrm{~A}$ & $148: 346$ & $1353 \pm 66$ & & $845.2 \pm 8.5$ & A.D. 597 \\
\hline-73 & $161: 169$ & $725 \pm 172$ & & $913.7 \pm 21.5$ & A.D. 1225 \\
\hline-75 & $161: 166$ & $32,660 \pm 1300$ & & $17.2 \pm 160.2$ & 30,710 B.C. \\
\hline
\end{tabular}




\begin{tabular}{|c|c|c|c|c|c|}
\hline $\begin{array}{c}\text { Laboratory } \\
\text { number }\end{array}$ & $\begin{array}{c}\text { Published } \\
\text { reference }\end{array}$ & $\begin{array}{l}\text { Original date or } \\
\text { other value }\end{array}$ & $\begin{array}{l}\text { Corrected date } \\
\text { or other value }\end{array}$ & $\partial \mathrm{C}^{14}, \% 0$ & $\begin{array}{l}\text { A.D./ } \\
\text { B.C. date }\end{array}$ \\
\hline .76 & $161: 166$ & $17,290 \pm 436$ & & $116.2 \pm 54.4$ & 15,340 B.C. \\
\hline .77 & $148: 347$ & $1090 \pm 423^{9}$ & & $751.9 \pm 36.5$ & A.D. 860 \\
\hline-79 & $161: 169$ & $3280 \pm 212^{9}$ & & $572.6 \pm 26.4$ & 1330 B.C. \\
\hline-80 & $161: 169$ & $590 \pm 260^{9}$ & $5900 \pm 260 \mathrm{c}$ & $413.0 \pm 32.5$ & 3950 B.C. \\
\hline-81 & $161: 169$ & $7760 \pm 335^{9}$ & & $327.8 \pm 41.7$ & 5810 B.C. \\
\hline-83 & $161: 167$ & $14,780 \pm 192$ & & $158.9 \pm 24.1$ & 12,830 B.C. \\
\hline-84 & $161: 168$ & $4160 \pm 119$ & & $596.1 \pm 14.7$ & 2210 B.C. \\
\hline$-84 \mathrm{~A}$ & $161: 168$ & $3140 \pm 187$ & & $676.5 \pm 23.3$ & 1190 B.C. \\
\hline-85 & $161: 168$ & $9200 \pm 308$ & & $318.2 \pm 38.4$ & 7250 B.C. \\
\hline$-85 A$ & $161: 168$ & $12,080 \pm 380$ & & $222.3 \pm 47.4$ & 10,130 B.C. \\
\hline-86 & $161: 168$ & $13,640 \pm 492$ & & $183.2 \pm 61.2$ & 11,690 B.C. \\
\hline .87 & $161: 171$ & $8310 \pm 267$ & & $355.5 \pm 32.6$ & 6360 B.C. \\
\hline-88 & $161: 171$ & $10,500 \pm 321$ & & $270.7 \pm 39.8$ & 8550 B.C. \\
\hline-90 & $161: 170$ & $12,820 \pm 444$ & & $202.8 \pm 55.4$ & 10,870 B.C. \\
\hline .91 & $161: 170$ & $14,160 \pm 483$ & & $171.6 \pm 59.8$ & 12,210 B.C. \\
\hline .92 & $161: 172$ & $\partial \mathrm{C}^{14}+3.4 \%$ & $3.4 \pm 0.9 \% \mathrm{c}$ & $1033.6 \pm 8.5$ & \\
\hline$-92 \mathrm{~A}$ & $161: 172$ & $970 \pm 79$ & & $886.7 \pm 9.8$ & A.D. 980 \\
\hline-94 & $161: 171$ & $8200 \pm 274$ & & $360.4 \pm 34.2$ & 6250 B.C. \\
\hline-95 & $161: 172$ & $10,810 \pm 361$ & & $260.4 \pm 44.9$ & 8860 B.C. \\
\hline-96 & $161: 171$ & $9740 \pm 189$ & & $297.4 \pm 22.9$ & 7790 B.C. \\
\hline-97 & $161: 171$ & $10,560 \pm 347$ & & $268.6 \pm 43.1$ & 8610 B.C. \\
\hline-98 & $161: 171$ & $10,650 \pm 354$ & & $265.5 \pm 44.2$ & 8700 B.C. \\
\hline-99 & $161: 172$ & $10,190 \pm 191$ & & $281.4 \pm 23.9$ & 8240 B.C. \\
\hline-101 & $161: 167$ & $3970 \pm 70$ & & $610.1 \pm 8.6$ & 2020 B.C. \\
\hline-102 & $161: 167$ & $19,620 \pm 372$ & & $87.0 \pm 46.3$ & 17,670 B.C. \\
\hline-103 & $161: 167$ & $209 \pm 91$ & & $974.3 \pm 11.4$ & A.D. 1741 \\
\hline-107 & $161: 169$ & $\partial \mathrm{C}^{14}+94 \%$ & $93.9 \pm 1.6 \% \mathrm{c}$ & $1938.8 \pm 16.3$ & \\
\hline-108 & $161: 169$ & $\partial \mathrm{C}^{14}+77 \%$ & $76.8 \pm 1.7 \%$ c & $1767.8 \pm 17.4$ & \\
\hline-109 & $161: 169$ & $\partial \mathrm{C}^{14}+25 \%$ & $24.7 \pm 1.1 \%$ c & $1246.5 \pm 10.6$ & \\
\hline-110 & $161: 167$ & $6550 \pm 134$ & & $442.5 \pm 16.8$ & 4600 B.C. \\
\hline-111 & $161: 170$ & $\partial \mathrm{C}^{14}+8 \%$ & $7.6 \pm 1.8 \% \mathrm{c}$ & $1076.2 \pm 17.7$ & \\
\hline-112 & $161: 171$ & $\partial \mathrm{C}^{14}+1 \%$ & $1.3 \pm 1.9 \% \mathrm{c}$ & $1012.7 \pm 18.9$ & \\
\hline-113 & $161: 171$ & $1070 \pm 156$ & & $875.5 \pm 19.4$ & A.D. 880 \\
\hline$-119^{7}$ & $161: 173$ & $18,911 \pm 407$ & & $95.0 \pm 50.6$ & 16,961 B.C. \\
\hline-122 & $161: 167$ & $3380 \pm 61$ & & $656.4 \pm 7.6$ & 1430 B.C. \\
\hline-123 & $161: 170$ & $\partial \mathrm{C}^{14}+5.3 \%$ & $5.6 \pm 1.9 \% \mathrm{c}$ & $1055.6 \pm 18.6$ & \\
\hline-125 & $161: 171$ & $\partial \mathrm{C}^{14}+3.5 \%$ & $3.5 \pm 1.9 \% \mathrm{c}$ & $1035.1 \pm 19.4$ & \\
\hline$-125 \mathrm{~A}$ & $161: 171$ & $\partial \mathrm{C}^{14}+2.3 \%$ & $2.2 \pm 1.9 \% \mathrm{c}$ & $1022.5 \pm 18.7$ & \\
\hline-126 & $161: 168$ & $10,654 \pm 188$ & & $265.5 \pm 23.2$ & 8704, B.C. \\
\hline-127 & $161: 171$ & $\partial \mathrm{C}^{14}+37.0 \%$ & $37.0 \pm 1.7 \% \mathrm{c}$ & $1370.4 \pm 16.7$ & \\
\hline-128 & $161: 171$ & $8390 \pm 240$ & & $352.0 \pm 31.1$ & 6440 B.C. \\
\hline-129 & $161: 171$ & $9835 \pm 309$ & & $294.0 \pm 38.4$ & 7885 B.C. \\
\hline-130 & $161: 171$ & $6110 \pm 129$ & & $467.3 \pm 15.8$ & 4160 B.C. \\
\hline-132 & $161: 171$ & $6240 \pm 233$ & & $460.0 \pm 29.0$ & 4290 B.C. \\
\hline-133 & $161: 171$ & $7025 \pm 274$ & & $417.1 \pm 34.2$ & 5075 B.C. \\
\hline-134 & $161: 171$ & $8150 \pm 304$ & & $362.5 \pm 37.9$ & 6200 B.C. \\
\hline-135 & $161: 171$ & $1560 \pm 168$ & & $823.1 \pm 20.9$ & A.D. 382 \\
\hline-136 & $161: 171$ & $5270 \pm 232$ & & $519.2 \pm 29.0$ & 3320 B.C. \\
\hline-137 & $161: 171$ & $1500 \pm 180$ & & $829.8 \pm 22.4$ & A.D. 450 \\
\hline-138 & $161: 171$ & $2630 \pm 190$ & & $721.1 \pm 23.7$ & 680 B.C. \\
\hline
\end{tabular}

${ }^{1}$ O-766, SI-3.

${ }^{2}$ SI-4, Y-1248; may equal W-597, W-598.

${ }^{3} \mathrm{~W}-136, \mathrm{~L}-433 \mathrm{C}$

${ }^{4} \mathrm{Y}-882$.

${ }^{5} \mathrm{Y}-843$.

${ }^{8} \mathrm{H}-72 / 88$.

${ }^{7}$ OWU-8.

$8 \mathrm{~W}-91$.

${ }^{\circ}$ Date shown incorporates a correction of -1200 years as an initial estimate of Paleozoic carbonate dilution.

${ }^{10}$ L221-B. 